Results We included 36 patients (age mean 50.8 (SD 11.2) years, male/female 30/6, body mass index median 29.6 (IQR 26.9$34.9) \mathrm{kg} / \mathrm{m}^{2}$, Epworth Sleepiness Scale 10.5 (4.6) points, oxygen desaturation index median 25.7 (16.0-49.1)/hour, apnoea-hypopnoea index median $28.1(19.0-57.0) /$ hour $)$. None of the patients reported skin discomfort, unpleasant tongue sensations or morning headache. There was no difference in patients' perceived sleep quality. There was a 59\% reduction in mouth dryness after active treatment compared to sham-stimulation. There were no severe adverse events (Table).

Conclusion TES of the UA dilator muscles in OSA can be delivered throughout the night with few side effects and does not lead to arousal from sleep, if appropriately titrated.

\section{Cough Sensation}

\section{S27 THE EFFECT OF P2X3 ANTAGONISM (AF-219) ON EXPERIMENTALLY EVOKED COUGH IN HEALTHY VOLUNTEERS AND CHRONIC COUGH PATIENTS}

\author{
${ }^{1} \mathrm{JA}$ Smith, ${ }^{2} \mathrm{M}$ Kitt, ${ }^{2} \mathrm{P}$ Butera, ${ }^{2} \mathrm{~A}$ Ford. ${ }^{1}$ University of Manchester, Manchester, UK; \\ ${ }^{2}$ Afferent Pharmaceuticals Inc., Sam Mateo, USA
}

\subsection{6/thoraxjnl-2016-209333.33}

Introduction and objectives Effective therapies for chronic cough are a significant unmet need. Recently a P2X3 antagonist (AF219), markedly reduced cough frequency in two phase 2 trials, but the role of P2X3 receptors and their ligand (adenosine triphosphate, ATP) in chronic cough is not well-understood. This study assessed the effect of P2X3 antagonism on cough evoked by capsaicin and ATP in healthy volunteers and chronic cough patients.

Method We performed a double-blind, placebo-controlled, randomised, 4-period crossover study. During each period $(\geq 48 \mathrm{~h}$ apart), cough challenges were performed $2 \mathrm{~h}$ after single doses of study medication [capsaicin $(0.48-1000 \mu \mathrm{M})$ periods $1 / 2$ and ATP $(0.227-929 \mu \mathrm{mol} / \mathrm{mL})$ periods 3/4]. Two cohorts were enrolled; cohort (1) 14 healthy volunteers (HV, mean age 37.5 yrs, 100\% male) and 12 chronic cough (CC, mean age 60.3 yrs, $17 \%$ male) received AF-219 300mg/placebo and cohort (2) 12 HV (mean age 34.8 yrs, 100\% male) and 12 CC (mean age 57.8 yrs, 25\% male) received AF-219 $50 \mathrm{mg} /$ placebo. Cough challenges consisted of four inhalations of each doubling concentration of tussive agent, from a dosimeter 30 s apart. Coughs in the first $15 \mathrm{~s}$ were counted and challenges continued to the maximum tolerated dose. The concentrations evoking at least 2 and 5 coughs, C2 and C5 (from inhalation 1) were analysed using mixed effect models; pharmacodynamic modelling was used to estimate Emax/ED50.

Results AF-219 had no effect on capsaicin C2 or C5 at $300 \mathrm{mg}$ or $50 \mathrm{mg}$ in HV or CC (all p > 0.05). For ATP challenges, AF$219300 \mathrm{mg}$ significantly increased C2 in CC (AF-219 10.8 $\mu \mathrm{mol} / \mathrm{mL}$ vs. placebo $2.3 \mu \mathrm{mol} / \mathrm{mL}, \mathrm{p}=0.005)$ but not $\mathrm{HV}$ $(\mathrm{p}=0.135)$, whereas AF-219 50mg significantly increased C2 in both groups (CC $40.4 \mu \mathrm{mol} / \mathrm{mL}$ vs. $2.8 \mu \mathrm{mol} / \mathrm{mL}, \mathrm{p}=0.002$ and $\mathrm{HV} 114.4 \mu \mathrm{mol} / \mathrm{mL}$ vs. $20.7 \mu \mathrm{mol} / \mathrm{mL}, \mathrm{p}=0.046)$. AF-219 had no significant effect on ATP C5 at either dose in HV (all $\mathrm{p}>0.05$ ), but in CC patients $50 \mathrm{mg}$ AF-219 (but not $300 \mathrm{mg}$ ) increased C5 $(70.1 \mu \mathrm{mol} / \mathrm{mL}$ vs. $17.1 \mu \mathrm{mol} / \mathrm{mL}, \mathrm{p}=0.027)$. Of note, ATP inhalation evoked less coughing than capsaicin, limiting the utility of the C5 endpoint.
Conclusions P2X3 antagonism reduced cough responses to ATP, particularly in patients with CC, but did not alter cough responses to an off-target tussive agent.

\section{S28 DETERMINANTS OF COUGH FREQUENCY IN ADULT HEALTHY VOLUNTEERS}

K Holt, C Gibbard, JA Smith. University of Manchester, Manchester, UK

\subsection{6/thoraxjnl-2016-209333.34}

Introduction Objective cough monitoring is a useful tool to investigate patterns of cough frequency and to evaluate novel cough treatments. The VitaloJAKTM (Vitalograph Ltd, UK) ambulatory cough monitor is a validated semi-automated system for the quantification of cough over 24 hours. Objective cough rates have yet to be quantified in large groups of healthy controls and the influences of subject factors are unclear.

Objective To assess objective cough frequency in a large group of healthy adults across a range of ages.

Method Objective 24 hour cough monitoring was performed using the VitaloJAKTM in adult healthy volunteers; those with a smoking history of $>20$ pack years and $<6$ months abstinence were excluded. The recordings were compressed using customwritten software and cough counted manually by trained cough counters and the daytime, night-time and total cough rates calculated. Daytime and total cough rates were log transformed for analysis. Independent t-tests (daytime and total) and Mann-Whitney $U$ test (night-time) assessed the effect of gender and previous smoking. Spearmans correlation coefficients evaluated the relationships between cough frequency, age, BMI, and pulmonary function.

Results Sixty healthy volunteers were recruited; 27 (45\%) males, median age 40 yrs (range 20-74), median $\mathrm{FEV}_{1}$ 103.0\% predicted (81-141), median FVC 105.5\% predicted (82-151), median BMI $24.6 \mathrm{~kg} / \mathrm{m} 2$ (16.8-39.8), 48 (80\%) of subjects had never smoked, median smoking history in the ex-smokers 2.9 (0.1-17) pack years. Median (IQR) $24 \mathrm{~h}$ cough rate was $0.17 \mathrm{c} / \mathrm{h}$ (0.05-0.87) with daytime rate of $0.26 \mathrm{c} / \mathrm{h}(0.63-1.35)$ and nighttime rate of $0.00 \mathrm{c} / \mathrm{h}(0.00-0.12)$. Males coughed significantly more than females over 24hours [median $0.42 \mathrm{c} / \mathrm{h}$ (IQR $0.13-$ $1.21)$ vs. $0.13 \mathrm{c} / \mathrm{h}(0.04-0.59), \mathrm{p}=0.038$ ] and during the day [0.37 c/h (0.11-1.42) vs. $0.19 \mathrm{c} / \mathrm{h}(0.0-0.91), \mathrm{p}=0.036]$, but not during the night $(\mathrm{p}=0.852)$. Cough frequency was not significantly correlated with age, BMI, $\mathrm{FEV}_{1}$ or FVC. Cough frequency was no different between never and ex-smokers for daytime or $24 \mathrm{~h}(\mathrm{p}=0.46$ and $\mathrm{p}=0.20)$ but overnight was slightly lower for ex- than never smokers [median $0.00 \mathrm{c} / \mathrm{h}$ (0.00-0.09) vs. $0.12 \mathrm{c} / \mathrm{h}(0.0-0.64), \mathrm{p}=0.037]$.

Conclusions In healthy adults, spontaneous cough frequency is unaffected by age, BMI, and pulmonary function. Interestingly, males coughed more frequently than females, in contrast to our current knowledge of gender differences in cough reflex sensitivity.

\section{S29 A RANDOMISED CONTROLLED TRIAL OF OVER THE COUNTER MEDICINE CS1002 FOR ACUTE COUGH}

${ }^{1} \mathrm{SS}$ Birring, ${ }^{2} \mathrm{~J}$ Brew, ${ }^{2} \mathrm{~T}$ Kilbourn, ${ }^{3} \mathrm{AH}$ Morice. ${ }^{1}$ Division of Asthma, Allergy and Lung Biology, King's College, London, UK; ${ }^{2}$ Infirst Healthcare Limited, London, UK; ${ }^{3}$ Hull York Medical School, Castle Hill Hospital, Hull, UK

10.1136/thoraxjnl-2016-209333.35 


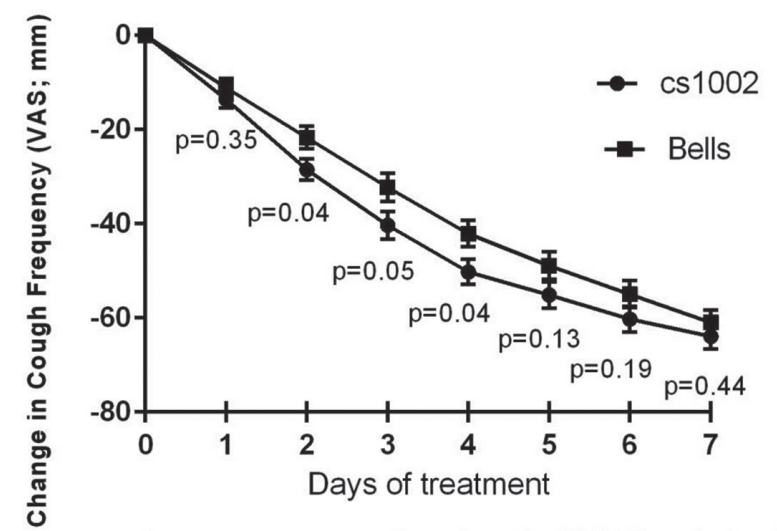

Least-square means based on the MMRM analysis \pm SE.

Abstract S29 Figure 1 Change in cough frequency over time (Full Analysis Set)

Introduction CS1002 contains diphenhydramine/ammoniumchloride/levomenthol in a demulcent preparation. We conducted a randomised controlled trial to compare the efficacy of CS1002 with Bell's Simple Linctus (BSL).

Methods 163 subjects with acute cough associated with upper respiratory tract infection, URTI (onset $\leq 7$ days and cough severity VAS $\geq 60 \mathrm{~mm}$ ), presenting to pharmacists (64\%) or general practitioners were randomised to CS1002 or BSL. The subjects (mean age 39 years, 57\% female) were instructed to take their medication four times daily $(5 / 5 / 10 / 10 \mathrm{ml})$ for 7 days or until resolution. Investigators were blinded to the treatment allocation. The primary analysis, cough severity VAS (0-100) was ANCOVA in an intention to treat population.

Results CS1002 ( $\mathrm{n}=82$ ) compared to BSL ( $\mathrm{n}=75)$, after 3 days, was associated with a greater reduction in cough severity (adjusted mean(SE) difference 5.9 (4.3), $\mathrm{p}=0.18$ ), cough sleep disruption VAS (mean diff $11.6(4.5), \mathrm{p}=0.01$ ) and cough frequency VAS (mean diff 8.1 (4.1), p =0.05, Figure 1). There was a greater improvement in QOL (LCQ-acute) with CS1002 compared to BSL after 5 days; mean diff $1.2(0.6), p=0.04$. The reduction in cough severity with CS1002 was similar in dry/

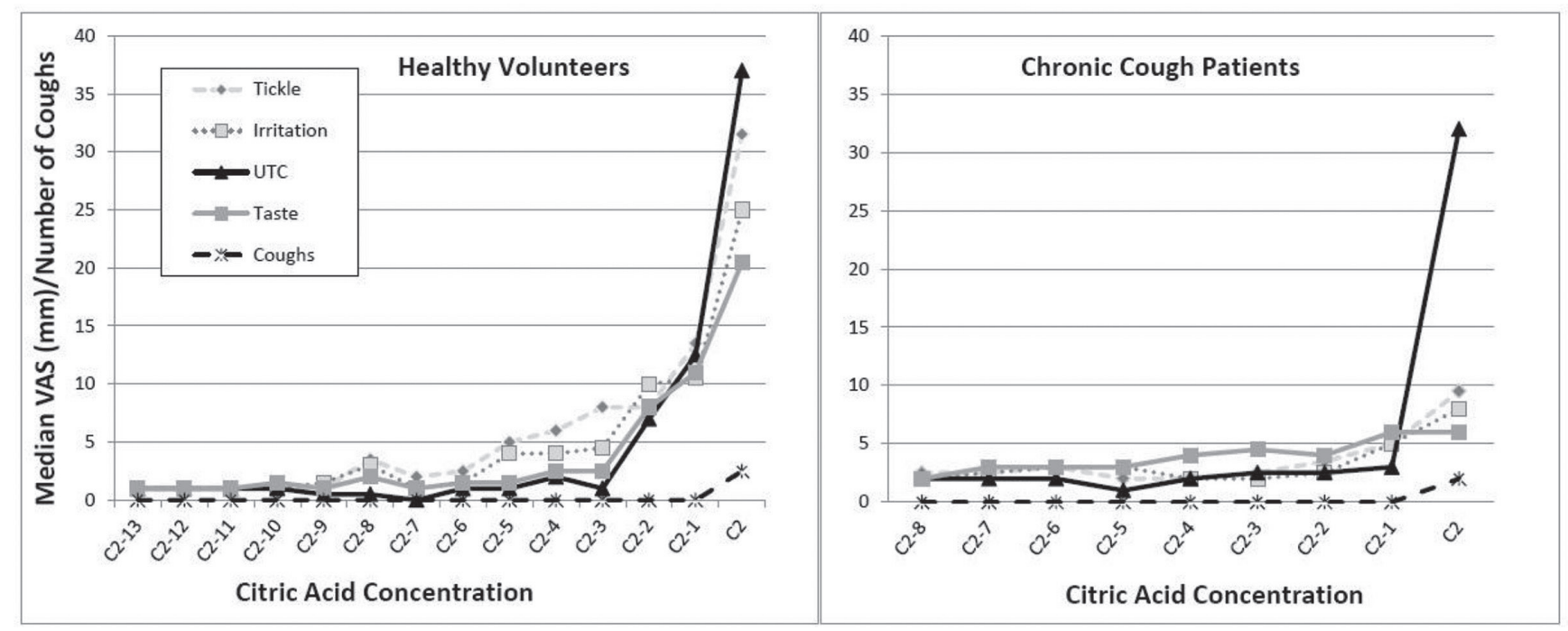

\section{Abstract S30 Figure 1}

chesty/tickly categories of cough. Cough resolved after 3 days in $24.4 \%$ of CS1002 subjects compared to $10.7 \%$ BSL, $\mathrm{p}=0.02$. CS1002 was well tolerated, similar to BSL.

In conclusion We have conducted the largest RCT in URTI with validated outcome measures. CS1002 reduced the impact of cough associated with URTI with a greater reduction in cough frequency and sleep disruption and improvement in QOL and cough resolution compared to BSL.

\section{S30 SENSATIONS ASSOCIATED WITH EXPERIMENTALLY EVOKED COUGH: A COMPARISON OF CHRONIC COUGH PATIENTS WITH HEALTHY CONTROLS}

IJ Mitchell, ${ }^{2} \mathrm{~B}$ Al-Sheklly, ${ }^{2} \mathrm{~B}$ Issa, ${ }^{1} \mathrm{~T}$ Collier, ${ }^{2} \mathrm{D}$ Corfield, ${ }^{2} \mathrm{JA}$ Smith. ${ }^{1}$ University Hospital of South Manchester, Manchester, UK; ${ }^{2}$ University of Manchester, Manchester, UK

\subsection{6/thoraxjnl-2016-209333.36}

Introduction and objectives Cough is generally considered a protective airway reflex, however emerging evidence suggests chronic coughing is provoked by noxious sensations from the airway and serves to relieve these sensations. We have found patients with unexplained chronic coughing identify sensations of irritation, tickle (throat) and the urge to cough (UTC) as important sensations provoking coughing. We hypothesised that inhaling low dose tussive agents would evoke similar sensations and could provide a model for investigating chronic cough.

Methods Twelve chronic cough patients (mean age $61.4 \mathrm{yrs}, 75 \%$ female, median cough duration 7 yrs) and 10 healthy volunteers (mean age 48.8 yrs, $40 \%$ female) inhaled increasing concentrations of citric acid from a dosimeter (0.01-4 M, 18 ascending concentrations). Following each inhalation subjects rated irritation, tickle, UTC and taste on $100 \mathrm{~mm}$ visual analogue scales (VAS; $0 \mathrm{~mm}=$ none and $100 \mathrm{~mm}=$ worst). The experiment continued until subjects coughed at least twice on any concentration of citric acid (C2). Somatosensory amplification score (SSAS) and State Trait Anxiety Index (STAI) were also collected. For the analysis, VAS data were aligned by the C2 concentration and sensation VAS scores compared using Mann-Whitney U tests. STAI scores were also compared with Mann-Whitney U tests and SSAS with an independent T test. 